\title{
Impact of body mass index on short-term outcomes after differentiated thyroid cancer surgery: a nationwide inpatient database study in Japan
}

\author{
Takaaki Konishi1,2, Michimasa Fujiogi2,3, Nobuaki Michihata4, Takayoshi Niwa1, Kojiro Morita2,5, Hiroki Matsui2, \\ Kiyohide Fushimi ${ }^{6}$, Masahiko Tanabe1, Yasuyuki Seto ${ }^{1}$ and Hideo Yasunaga ${ }^{2}$ \\ 'Department of Breast and Endocrine Surgery, Graduate School of Medicine, The University of Tokyo, Tokyo, Japan \\ 2Department of Clinical Epidemiology and Health Economics, School of Public Health, The University of Tokyo, Tokyo, Japan \\ 3Department of Emergency Medicine, Massachusetts General Hospital, Harvard Medical School, Boston, Massachusetts, USA \\ ${ }^{4}$ Department of Health Services Research, Graduate School of Medicine, The University of Tokyo, Tokyo, Japan \\ ${ }^{5}$ Department of Health Services, Faculty of Medicine, University of Tsukuba, Tsukuba, Ibaraki, Japan \\ ${ }^{6}$ Department of Health Policy and Informatics, Tokyo Medical and Dental University Graduate School, Tokyo, Japan \\ Correspondence should be addressed to T Konishi: takaakonishi-ncd@umin.ac.jp
}

\begin{abstract}
Introduction: Recent studies have shown worse post-operative outcomes following several surgeries in underweight or obese patients. However, the association between body mass index (BMI) and short-term outcomes following thyroid cancer surgery remains unclear because of the small number of patients, deficits in background data known as risk factors (e.g. cancer stage, operative procedure, intraoperative device use and hospital volume) and categorisation of BMI.

Methods: We identified patients who underwent thyroidectomy for differentiated thyroid cancer from July 2010 to March 2017 using a Japanese nationwide inpatient database. We used restricted cubic spline (RCS) analyses to investigate potential nonlinear associations between BMI (without categorisation) and outcomes: post-operative complications (local and general), duration of anaesthesia, post-operative length of hospital stay and hospitalisation costs. The analyses were adjusted for demographic and clinical backgrounds including the above-stated factors. We also performed multivariable regression analyses for the outcomes with categorisation of BMI.

Results: Among 59,671 eligible patients, the median BMI was $22.9 \mathrm{~kg} / \mathrm{m}^{2}$ (interquartile range (IQR), $20.7-25.6 \mathrm{~kg} / \mathrm{m}^{2}$ ). In total, 3860 patients $(6.5 \%)$ had local complications and 787 patients $(1.3 \%)$ had general complications. Although there were no significant associations with local complications, such as bleeding, recurrent laryngeal nerve paralysis and surgical site infection, the occurrence of general complications was significantly associated with higher BMI. BMI showed a linear association with the duration of anaesthesia and U-shaped associations with post-operative length of stay and hospitalisation costs. The lowest points of the U-shaped curves occurred at a BMI of approximately $24 \mathrm{~kg} / \mathrm{m}^{2}$. The multivariate regression analyses showed consistent results with the RCS analyses.
\end{abstract}


Discussion/conclusion: Whereas RCS analyses revealed no significant associations

between BMI and post-operative local complications, obesity was significantly associated

with the occurrence of general complications. The linear association between BMI and

duration of anaesthesia corresponds to previous studies. Although post-operative length

of stay and total hospitalisation costs demonstrated U-shaped associations, the slight

differences would not be clinically important. Even if surgeons must pay attention to

general complications in obese patients undergoing thyroid cancer surgery as well as

other surgeries, underweight and overweight patients can undergo thyroidectomy as

safely as patients with normal BMI.

\section{Introduction}

Thyroidectomy is the only radical treatment for thyroid cancer. Several complications may occur following thyroidectomy: for example, post-operative bleeding, recurrent laryngeal nerve (RLN) paralysis, post-operative hypocalcaemia and chylothorax $(1,2,3,4,5,6)$. Postoperative bleeding can be fatal because of subsequent laryngeal oedema and airway constriction; and some factors such as Graves' disease, active antiplatelet or anticoagulant medication, chronic renal disease, partial thyroidectomy and inflammatory thyroid conditions are known risk factors $(7,8)$. RLN paralysis occurs in 3-5\% of people after thyroid surgery. Although older age, thyrotoxicosis, extended surgery and low-volume hospital are risk factors for RLN paralysis $(9,10)$, intraoperative device use, such as nerve monitoring and energy devices, can be associated with a low occurrence of RLN paralysis $(4,11)$.

Whereas body mass index (BMI) is associated with short-term outcomes following a variety of procedures such as abdominal and breast surgery $(12,13,14,15,16$, 17), the association between BMI and short-term outcomes (e.g. post-operative complications) following thyroid cancer surgery is controversial. A US study involving 18,825 patients who underwent thyroidectomy reported that BMI $>25 \mathrm{~kg} / \mathrm{m}^{2}$ was significantly associated with higher morbidity and longer operative time, but the study did not include RLN paralysis as the outcome, and the results were not adjusted for cancer stage or most of the above-stated factors (18). Other studies in France and Italy showed no significant differences in post-operative complications among different BMI categories despite longer operative time in patients with BMI $>25 \mathrm{~kg} / \mathrm{m}^{2}$ than in those with normal BMI $(19,20,21)$. However, because these studies had a relatively small number of cases, the analyses lacked adjustment for the patients' backgrounds. Furthermore, these studies categorised BMI, which resulted in a loss of information and lower statistical power compared with a restricted cubic spline (RCS) analysis $(22,23)$.

Therefore, the present study was performed to investigate the association between short-term surgical outcomes and BMI in patients undergoing thyroidectomy for thyroid cancer with an RCS analysis using a nationwide inpatient database in Japan.

\section{Materials and methods}

\section{Database}

This nationwide retrospective cohort study was performed using the Diagnosis Procedure Combination database. The database included hospital administrative claims data and discharge abstracts for approximately 8,000,000 inpatients in more than 1200 hospitals throughout Japan, covering approximately half of all inpatient admissions to acute care hospitals in Japan (24). All 82 university hospitals were obliged to participate in the database, whereas participation by community hospitals was voluntary.

The database included the following data: unique hospital identifiers; patients' age and BMI at admission; sex; smoking history; main diagnoses and comorbidities at admission and complications after admission recorded with text data in the Japanese language and the International Classification of Diseases, Tenth Revision (ICD-10) codes; clinical tumour $(\mathrm{T})$, node $(\mathrm{N})$ and metastasis classification of malignant tumours; interventional/surgical procedures indexed by the original Japanese codes; duration of anaesthesia; length of stay and total hospitalisation cost. The total hospitalisation cost was calculated according to reference prices in the fee schedule that determine itemby-item prices for inpatient services, such as operations. All discharge abstract data for each patient were recorded at discharge by the attending physicians. A previous
This work is licensed under a Creative Commons Attribution-NonCommercial 4.0 International License. Bioscientifica.com at 04/26/2023 10:40:10AM 
validation study showed good sensitivity and specificity of the diagnoses and procedure records in the database (25).

The need for informed consent in the present study was waived because of the anonymity of the patient database. Study approval was obtained from the Institutional Review Board at The University of Tokyo (approval number: 3501(3) (25 December 2017)).

\section{Study protocol}

We identified patients with thyroid cancer who underwent thyroidectomy from July 2010 to March 2017. We used the Japanese original procedure codes for surgery to identify patients who underwent the procedures. We excluded patients who (i) were $<18$ years old, (ii) were not preoperatively diagnosed with differentiated thyroid cancer (i.e. poorly differentiated thyroid carcinoma, anaplastic thyroid carcinoma or malignant lymphoma), (iii) had distant metastasis (these patients were excluded because they could be malnourished because of cachexia), (iv) underwent another operation for conditions other than complications of thyroidectomy during the hospitalisation and (v) had outlier data for their height $(<100 \mathrm{~cm}$ or $>200 \mathrm{~cm})$ or weight $(<20 \mathrm{~kg}$ or $>200 \mathrm{~kg})$.

We categorised the eligible patients into five groups according to their BMI and compared their outcomes: $<18.5 \mathrm{~kg} / \mathrm{m}^{2}$ (underweight), $18.5-21.9 \mathrm{~kg} / \mathrm{m}^{2}$ (low-normal weight), $22.0-24.9 \mathrm{~kg} / \mathrm{m}^{2}$ (high-normal weight), 25.0-29.9 $\mathrm{kg} / \mathrm{m}^{2}$ (overweight) and $\geq 30.0 \mathrm{~kg} / \mathrm{m}^{2}$ (obese).

The primary outcome was the occurrence of postoperative complications, which were divided into local and general complications. We defined local complications as bleeding, RLN paralysis (temporary or permanent), surgical site infection, chyle leakage (including chylothorax) and oesophageal injury. The general complications were respiratory complications, urinary tract infection, sepsis, heart failure, stroke, acute renal failure and pulmonary embolism. The definitions of these terms are listed in Supplementary Table 1 (see section on supplementary materials given at the end of this article).

The secondary outcomes were prescriptions for hypocalcaemia, duration of anaesthesia, post-operative length of stay and total hospitalisation costs. We did not define prescriptions for hypocalcaemia as complications because prophylactic medication to prevent symptomatic hypocalcaemia is commonly prescribed in Japan $(26,27)$, and the database we used does not include data that would enable us to distinguish prophylaxis from treatment. We defined the currency exchange rate as 110 Japanese yen per 1 US dollar.

\section{Statistical analysis}

We examined the following patient background factors: sex, age, smoking history (past/current smoker), comorbidities, preoperative anticoagulant use, preoperative diagnosis, clinical $\mathrm{T}$ and $\mathrm{N}$ classification (recorded at admission), operative procedure, extended lymph node dissection, intraoperative device use (energy devices and neuromonitoring), type of hospital (teaching hospital) and hospital volume. Age was categorised into four groups: $<55,55-64,65-74$ and $\geq 75$ years. Comorbidities were assessed with the Charlson comorbidity index (28) and categorised into three groups: 2,3 and $\geq 4$. Preoperative diagnoses were categorised as papillary, follicular, medullary and unspecified carcinoma. They were categorised on the basis of diagnoses recorded on admission, not on the results of preoperative investigations, which are not included in the database used in this study. Operative procedures were categorised as total and hemi/partial thyroidectomy. Because the database does not include information on whether central node dissection was performed, Japanese original procedure codes were used to define extended node dissection as dissection that extended more than the central lymph nodes. In Japan, the surgical procedure is generally chosen in accordance with the clinical practice guideline published by the Japan Associations of Endocrine Surgeons (29). In this guideline, hemi/partial thyroidectomy plus prophylactic central dissection is recommended for low- and intermediate-risk papillary carcinoma and total thyroidectomy plus extended dissection for high-risk papillary carcinoma. Hemi/partial or total thyroidectomy without dissection is recommended for follicular carcinoma, depending on metastasis, extent of invasion, and other risk factors. Hemi/partial or total thyroidectomy plus prophylactic central dissection is recommended for medullary carcinoma, depending on RET gene mutation. Hospital volume was defined as the number of eligible thyroidectomies performed annually at each hospital and was categorised into tertiles (low-, medium- and high-volume) with approximately equal numbers of patients in each group. These background factors were adjusted for in the following two analyses.

First, we performed multivariable regression analysis among the five BMI groups using a generalised estimating equation: logistic regression analyses for primary outcomes (i.e. post-operative complications and its subdivisions) and linear regression analyses for secondary outcomes (i.e. duration of anaesthesia, post-operative length of stay and total hospitalisation cost). The generalised estimating

This work is licensed under a Creative Commons Attribution-NonCommercial 4.0 International License Bioscientifica.com at 04/26/2023 10:40:10AM 
equation enabled us to adjust for within-hospital clustering of variables, such as patient characteristics or physician practice patterns within the same hospital. The explanatory variables were the above-mentioned patient background factors. We calculated odds ratios and 95\% CIs for each BMI group with respect to the reference group of low-normal BMI.

Secondly, we conducted RCS functions to investigate the non-linear association between BMI and outcomes. Whereas ordinally regression analysis with categorised continuous BMI measurements can lose information and power, all data points were used to estimate the doseresponse association between a continuous variable and outcomes in an RCS analysis $(22,23)$. The splines were restricted to linear below the first-knot point and above the last-knot point. In this study, we used four points $\left(18.5,22.0,25.0\right.$ and $\left.30.0 \mathrm{~kg} / \mathrm{m}^{2}\right)$ as the knots in the cubic splines to allow for non-linear effects of continuous BMI measurement. We also fitted the generalised estimating equation to the RCS analyses, as in the regression analyses, and calculated the odds ratios and 95\% CIs for each BMI value with respect to the reference value of $22.0 \mathrm{~kg} / \mathrm{m}^{2}$. The spline curve was constructed using the 'xbrcspline' and 'mkspline' commands in Stata (StataCorp, College Station, TX).

All hypothesis tests had a two-sided significance level of 0.05 , and all statistical analyses were conducted using Stata/MP 16.0 (StataCorp).

\section{Results}

In total, 64,549 patients who underwent thyroid cancer surgery from July 2010 to March 2017 were identified. We excluded 4878 patients who met the following exclusion criteria: (i) <18 years old ( $n=353$ ), (ii) not preoperatively diagnosed with differentiated thyroid cancer $(n=706)$, (iii) with distant metastasis ( $n=1652$ ), (iv) underwent another operation for conditions other than complications of thyroidectomy during the hospitalisation $(n=1738)$ and $(v)$ had outlier data for height or weight $(n=429)$. Of the 59,671 eligible patients, the underweight group comprised 4344 patients $(7.3 \%)$, the low-normal weight group comprised 19,242 patients (32\%), the high-normal weight group comprised 18,444 patients (31\%), the overweight group comprised 13,928 patients (23\%) and the obese group comprised 3713 patients (6.2\%). Overall, the median BMI was $22.9 \mathrm{~kg} / \mathrm{m}^{2}$ (interquartile range (IQR), 20.7-25.6 kg/m²).

Table 1 shows the baseline characteristics of all patients $(n=59,671)$ and of the groups categorised by BMI. In this study, 14,697 patients (25\%) were male, and approximately half of the patients were $<55$ years old (44\%), diagnosed with papillary carcinoma (44\%), had T1 (43\%) and N1 (53\%) cancer and underwent total thyroidectomy (48\%). Patients in the underweight and low-normal weight groups were more likely to be female than patients in the other groups. Patients in the obese group were more likely to have comorbidities and advanced $\mathrm{T} / \mathrm{N}$ classification and to undergo total thyroidectomy.

Table 2 shows the crude outcomes of all patients and of the groups categorised by BMI. In total, 4497 patients (7.5\%) had post-operative complications: 3860 patients (6.5\%) had local complications, namely bleeding (1.8\%), RLN paralysis (temporary or permanent) (3.2\%) and surgical site infection (1.3\%); 787 patients (1.3\%) had general complications. Additionally, 13,960 patients (22.9\%) received prophylactic or therapeutic prescriptions for hypocalcaemia. The median duration of anaesthesia, post-operative length of stay and total hospitalisation costs were $190 \mathrm{~min}$ (IQR, 143-258 min), 6 days (IQR, 4-8 days) and 6408 US dollars (IQR, 5649-7480 US dollars), respectively. Patients in the overweight and obese group showed a higher occurrence of complication, longer duration of anaesthesia and higher total hospitalisation costs than patients in the other groups.

The results of the multivariable regression analyses are shown in Table 3. Although there were no significant differences in post-operative complications among the different BMI categories except for surgical site infection for the high-normal group and general complications for the obese group (each: $P=0.04$ ), secondary outcomes showed significant differences. Patients in the high-normal, overweight and obese groups had a significantly longer duration of anaesthesia with reference to those in the lownormal group (all $P<0.001$ ). Patients in the underweight and obese groups had significantly longer stays and higher costs, whereas patients in the high-normal group had significantly shorter stays and lower costs, with reference to those in the low-normal group. Multivariable analysis for prescriptions for hypocalcaemia was conducted without a generalised estimated equation because of estimated divergence; this showed no significant differences between the BMI categories.

Figures illustrates the adjusted dose-response associations between BMI and the dependent variables in the RCS analyses. There were no significant doseresponse associations for postoperative complications, local complications, bleeding, RLN paralysis or surgical site infection (Fig. 1A, B, C, D and E). A J-shaped association was shown between BMI and the occurrence of general 


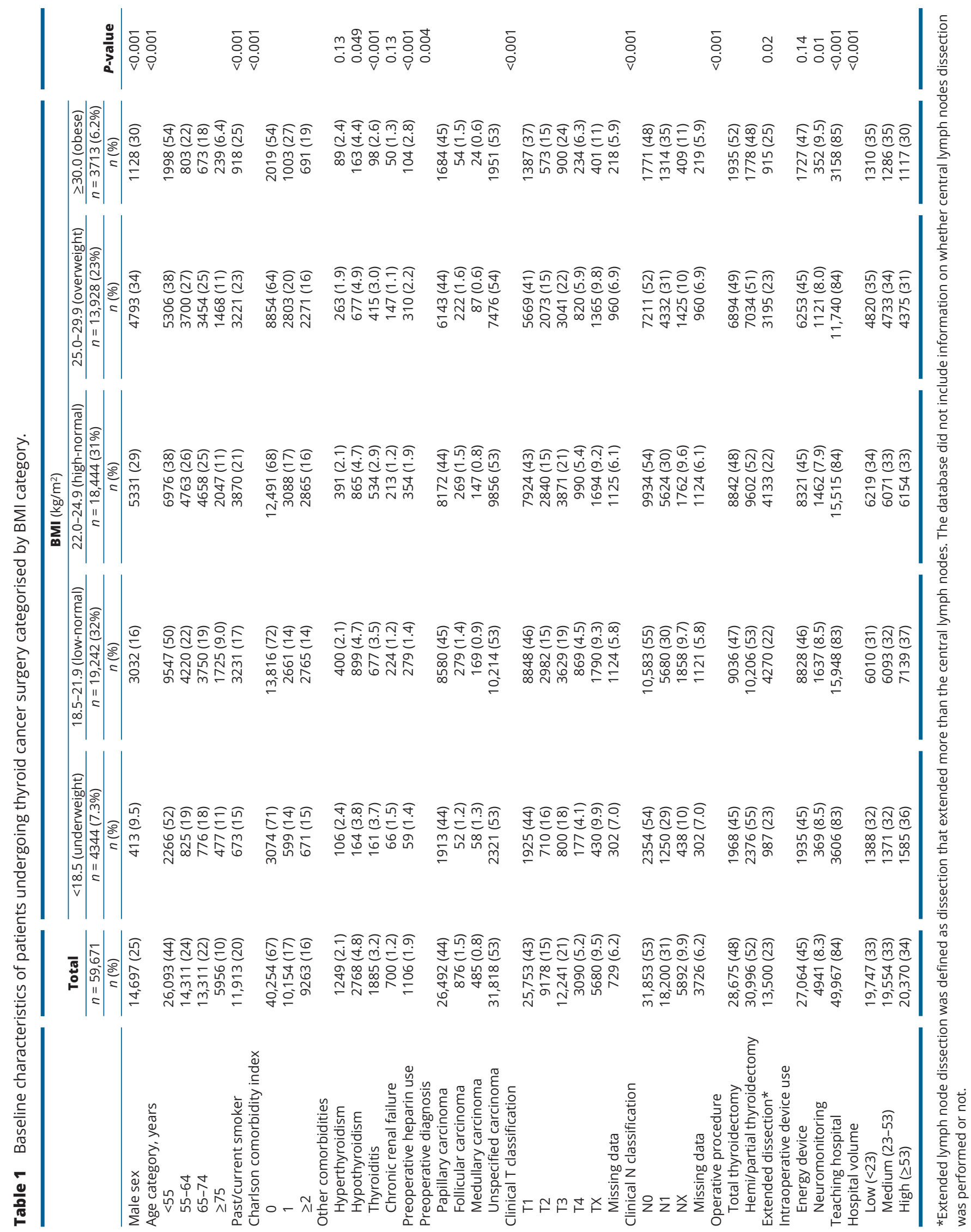




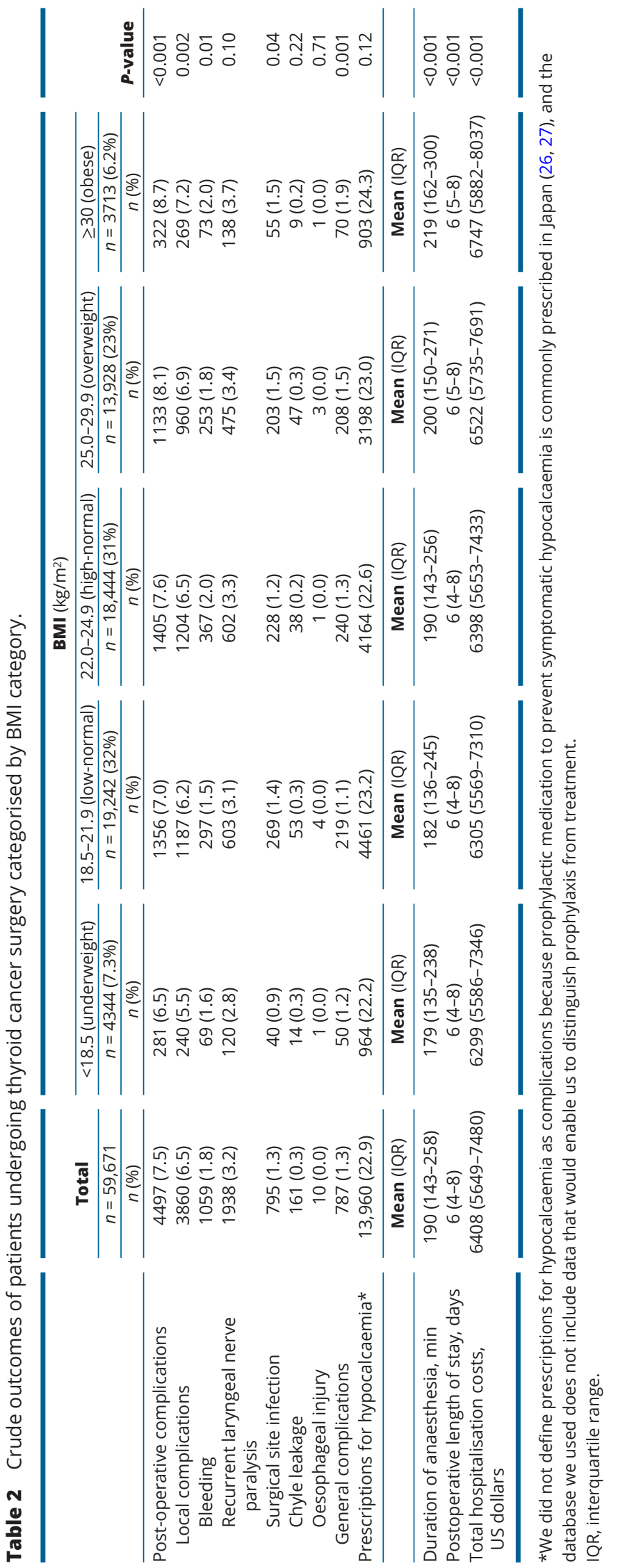

https://etj.bioscientifica.com https://doi.org/10.1530/ETJ-21-0081 (c) 2022 The authors Published by Bioscientifica Ltd. complications (Fig. 1F), a linear association was shown between BMI and duration of anaesthesia (Fig. 1G) and U-shaped associations were observed between BMI and post-operative length of stay or total hospitalisation costs. The lowest points of the U-shaped curves occurred at a BMI of approximately $24 \mathrm{~kg} / \mathrm{m}^{2}$ (Fig. $1 \mathrm{H}$ and I). The results of all RCS analyses were compatible with those of the logistic or linear regression analyses. Regarding prescriptions for hypocalcaemia, RCS was conducted without a generalised estimated equation; the results are shown in Supplementary Fig. 1.

\section{Discussion}

In this study, we investigated the association between BMI and short-term outcomes after thyroid cancer surgery using a nationwide inpatient database in Japan. The RCS analyses showed no significant associations of BMI with local complications (including bleeding, RLN paralysis and surgical site infection) but demonstrated a J-shaped association of BMI with the occurrence of general complications, a linear association with duration of anaesthesia and U-shaped associations with post-operative length of stay and total hospitalisation costs. The results of the multivariate regression analyses were similar to the results of the RCS analyses. Consequently, we consider that both underweight and overweight patients with thyroid cancer can undergo surgery as safely as patients with normal BMI, and clinicians must pay attention to general complications in obese patients.

Previous studies have demonstrated discordant results regarding the association between BMI and short-term outcomes following thyroid cancer surgery $(18,19,20$, 21), presumably because these studies failed to adjust for certain patients' background characteristics (e.g. cancer stage, intraoperative device use and some comorbidities). Furthermore, these studies conducted analyses with continuous BMI categorisation in patients with thyroid cancer, providing less information and power than RCS analyses $(22,23)$. Therefore, we conducted RCS analyses with adjustment for the above-stated background factors, as in previous studies evaluating other surgeries $(15,17)$.

In the present study, $29 \%$ of the patients had a BMI $>25.0 \mathrm{~kg} / \mathrm{m}^{2}$ (overweight and obese), which is considerably lower than that of previous studies reported in Western countries. For example, the proportion of such patients was $71 \%$ in a study from the United States (18) and 54\% in a study from France (21). This discrepancy is plausible because BMI distribution differs between 


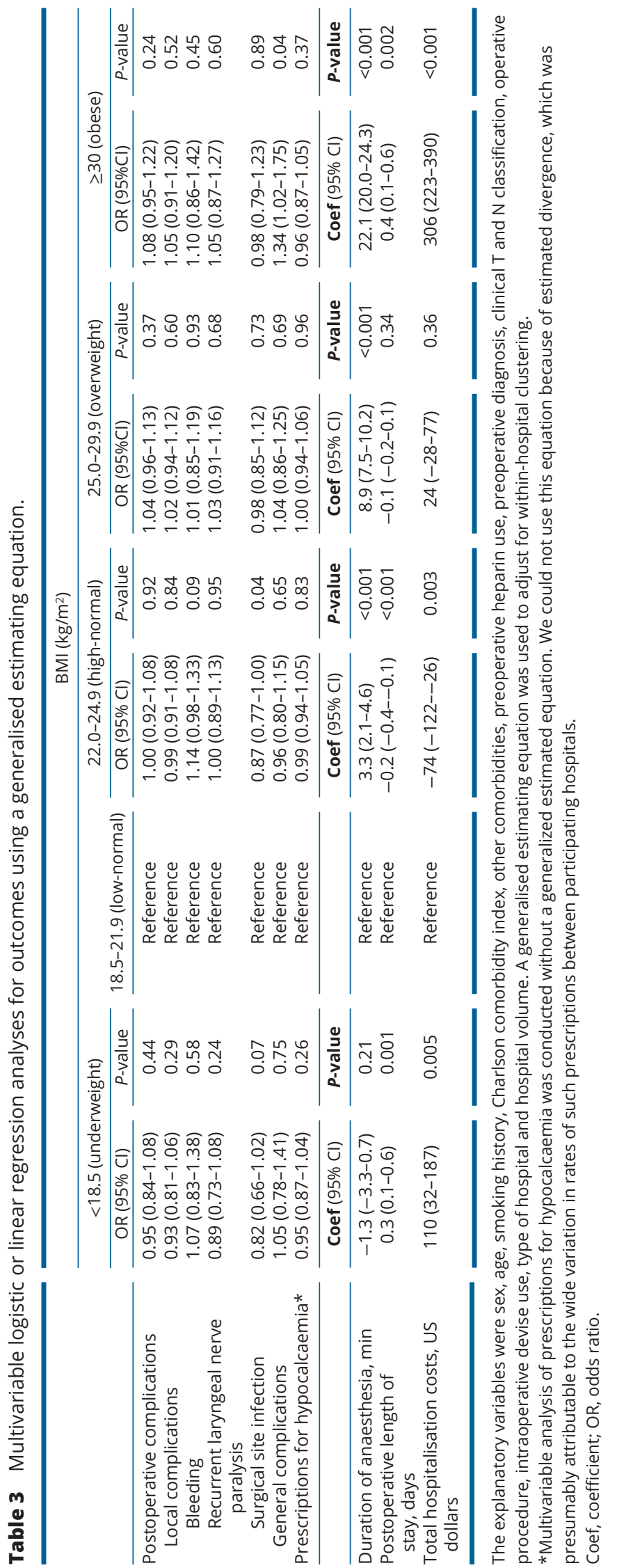

https://etj.bioscientifica.com https://doi.org/10.1530/ETJ-21-0081
(C) 2022 The authors Published by Bioscientifica Ltd.
Western and Asian populations (30,31). Moreover, we were able to analyse an underweight group, and such patients were rarely addressed in previous studies because of an inadequate number of patients for analysis.

The present study showed no significant associations of high BMI with the occurrence of total and local complications. However, these results do not correspond with the previous US study, which showed an association with high BMI and the occurrence of total complications and wound complications (18). In other surgeries (e.g. abdominal and breast surgery), high BMI is associated with the occurrence of post-operative complications because overweight and obese patients are likely to develop complications $(12,13,14,15,16,17)$. However, because thyroidectomy is a superficial procedure with a relatively small incision and rarely involves local invasiveness and inflammatory response, the current analyses showed that high BMI was not associated with the occurrence of surgical site infection. Additionally, because the s.c. and visceral adipose tissue of the neck would be thinner than that of the trunk, high BMI might not affect the difficulty of thyroidectomy; that is, it would not be difficult to identify vessels, nerves and lymph nodes, and to stop bleeding in neck adipose tissue, even in obese patients. Indeed, the RCS analyses also demonstrated no significant associations of high BMI with the occurrence of bleeding and RLN paralysis. Nonetheless, the current analysis showed that obese patients undergoing thyroidectomy were likely to develop general complications. According to our study (Supplementary Table 2), high BMI appears to be associated mainly with the occurrence of respiratory complications and heart failure, as well as in abdominal surgeries $(12,15)$. This is plausible because obesity is a known risk factor for these events $(32,33)$, and these may occur occasionally in obese patients even after the relatively minimal invasiveness of thyroidectomy.

This study also showed no significant associations between low BMI and post-operative complications. We previously found no significant association between low BMI and post-operative complications in breast cancer surgery, unlike in abdominal surgery (17). Whereas underweight patients might not withstand surgeryinduced stress and fasting in abdominal surgery, stress and 1-day abstinence from food prior to breast and thyroid cancer surgery may not substantially affect post-operative complications.

The RCS analysis showed a linear association between BMI and duration of anaesthesia, similar to findings in previous studies $(18,19)$. The authors in these studies stated that this association tendency resulted from 


\section{European Thyroid JOURNAL}
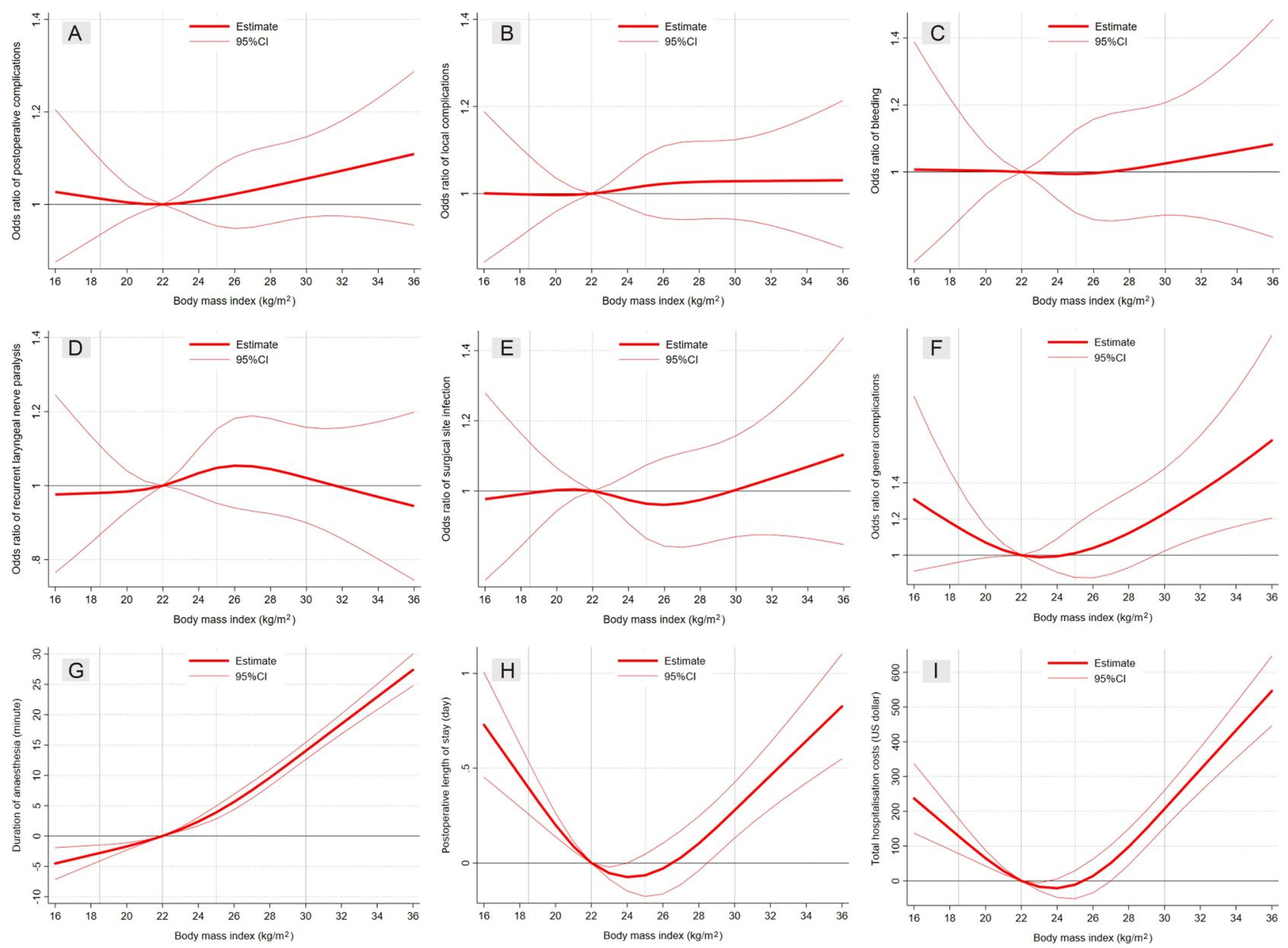

Figure 1

Adjusted dose-response association between in-hospital outcomes and BMI according to multivariable logistic (or linear regression) analysis using a restricted cubic spline with four knots $\left(18.5,22.0,25.0\right.$ and $\left.30.0 \mathrm{~kg} / \mathrm{m}^{2}\right)$. On the $Y$-axis is the odds ratio (or differences) for outcomes, comparing individuals with a BMI of $22 \mathrm{~kg} / \mathrm{m}^{2}$ and those with any other BMI. Estimates are shown with $95 \% \mathrm{Cls}$. The points of intersection of the lines on the X-axis and the restricted cubic spline represent the four knots $\left(18.5,22.0,25.0\right.$ and $\left.30.0 \mathrm{~kg} / \mathrm{m}^{2}\right)$. (A) Post-operative complications, (B) local complications, (C) bleeding, (D) recurrent laryngeal nerve paralysis, (E) surgical site infection, (F) general complications, $(G)$ duration of anaesthesia, (H) post-operative length of stay and (I) total hospitalisation costs.

technical challenges during operation (e.g. identifying the parathyroid) and difficulty in positioning and general anaesthesia (e.g. airway management (33) and drug pharmacokinetics differences (34)). Because longer duration of operation and anaesthesia are generally considered to be associated with worse post-operative complications (35), obesity would be significantly associated with the occurrence of general complications.

Post-operative length of stay and total hospitalisation costs demonstrated U-shaped associations with BMI, and the lowest points occurred at a BMI of approximately 24 $\mathrm{kg} / \mathrm{m}^{2}$, which suggests an 'obesity paradox' indicating improved outcomes with mild obesity $(13,15,36)$. However, the difference in the post-operative length of stay was less than 1 day. Furthermore, whereas the post-operative length of stay in the present study is comparable with that of previous studies reported from Japan $(37,38,39)$, it is considerably longer than that of previous studies from other countries. This is presumably because the length of stay in Japan is generally long, being approximately three times that in Western countries (40), this difference reportedly being attributable to differences in health care systems (41). Additionally, both clinicians and patients in Japan prefer to closely monitor surgical wounds and laboratory findings in a hospital setting. Therefore, the difference in hospital stay would be unimportant from a clinical standpoint other than its effect on increased hospitalisation costs.

Post-operative

hypocalcaemia and hypoparathyroidism are major complications specific to 
thyroidectomy $(1,2,3)$. Previous studies have found no significant associations between BMI and post-operative hypoparathyroidism $(20,21)$. In the current cohort, some patients would have been diagnosed as having hypocalcaemia or hypoparathyroidism to enable obtain prophylactic prescriptions; in Japan, calcium and/or vitamin D supplements are commonly administered prophylactically to prevent symptomatic hypocalcaemia $(26,27)$. Indeed, the current proportion of prescriptions for hypocalcaemia (22.9\%) was higher than the occurrence of hypocalcaemia $(11.6 \%)$ reported in a recent Japanese study (42). Unfortunately, the database we used does not provide data that would have enabled us to investigate the severity of hypocalcaemia and distinguish between prophylaxis and treatment (4). We therefore could not determine the actual occurrence of the complication. Furthermore, a generalised estimated equation to adjust for withinhospital clustering could not be adopted in multivariable analyses for prescriptions because of divergence, which was presumably attributable to substantial variations in prophylactic prescriptions between hospitals. However, the current analyses demonstrated no significant association between these prescriptions and BMI, indicating that BMI did not impact clinical practices regarding hypocalcaemia and hypoparathyroidism.

Several limitations of this study should be acknowledged. First, we were unable to assess some details that are specific to thyroidectomy and thyroid cancer. For example, because the database used in this study does not contain laboratory data, including serum calcium or symptoms, we were not able to distinguish between prophylaxis and treatment for hypocalcaemia. Additionally, oncological data, such as serum concentrations of thyroglobulin, calcitonin and carcinoembryonic antigen, are not listed in the database and therefore could not be evaluated. Secondly, the database does not provide detailed surgical information, such as operative time and surgeons' individual surgical skill levels. We also assessed duration of anaesthesia instead of operative time. Furthermore, because surgeons' skill can affect post-operative outcomes, we used hospital type (teaching or non-teaching hospital) and hospital volume as explanatory variables to adjust for the surgeons' expertise. Finally, we could not distinguish whether a particular patient's low BMI was a result of malnutrition owing to illness or a healthy condition without malnutrition; however, this is hardly distinguishable even clinically.

In summary, we assessed the association between BMI and the short-term surgical outcomes of differentiated thyroidcancer with adjustment for the patients' backgrounds using a nationwide inpatient database in Japan. The RCS analyses displayed no significant associations between BMI and post-operative complications, namely bleeding, RLN paralysis and surgical site infection. Obesity was significantly associated with the occurrence of general complications. Furthermore, the RCS analyses demonstrated a linear association for duration of anaesthesia and U-shaped associations for post-operative length of stay and total hospitalisation costs. Therefore, patients with thyroid cancer can safely undergo surgery regardless of BMI, while considering general complications in obese patients.

\section{Supplementary materials}

This is linked to the online version of the paper at https://doi.org/10.1530/ ETJ-21-0081.

\section{Declaration of interest}

The authors declare that there is no conflict of interest that could be perceived as prejudicing the impartiality of the research reported.

\section{Funding}

This work was supported by grants from the Ministry of Health, Labour and Welfare, Japan (19AA2007 and 20AA2005) and the Ministry of Education, Culture, Sports, Science and Technology, Japan (20H03907).

\section{Statement of ethics}

The need for informed consent in the present study was waived because of the anonymity of the patient database. Study approval was obtained from the Institutional Review Board at The University of Tokyo.

\section{Author contribution statement}

$T K, M F$, and $T N$ conceived the study concept and study design. $K M, H M$, and $\mathrm{K} F$ performed compilation and synthesis of the data. T K, M F, and $\mathrm{N}$ $M$ carried out statistical analysis. T K drafted the manuscript and M F, N M, $\mathrm{T} N, \mathrm{M} \mathrm{T}$, and $\mathrm{H} Y$ revised it. $\mathrm{Y} S$ and $\mathrm{H} \mathrm{Y}$ supervised the research project. All authors participated in interpretation of the results and writing of the report, and approved the final version of this manuscript.

\section{References}

1 Rosato L, Avenia N, Bernante P, De Palma M, Gulino G, Nasi PG, Pelizzo MR \& Pezzullo L. Complications of thyroid surgery: analysis of a multicentric study on 14,934 patients operated on in Italy over 5 years. World Journal of Surgery 200428 271-276. (https://doi. org/10.1007/s00268-003-6903-1)

2 Filho JG \& Kowalski LP. Postoperative complications of thyroidectomy for differentiated thyroid carcinoma. American Journal of Otolaryngology 200425 225-230. (https://doi.org/10.1016/j.amjoto.2004.02.001)

3 Lee YS, Nam KH, Chung WY, Chang HS \& Park CS. Postoperative complications of thyroid cancer in a single center experience. Journal 
of Korean Medical Science 201025 541-545. (https://doi.org/10.3346/ jkms.2010.25.4.541)

4 Konishi T, Fujiogi M, Niwa T, Morita K, Matsui H, Fushimi K, Tanabe M, Seto Y \& Yasunaga H. Comparison of outcomes after differentiated thyroid cancer surgery performed with and without energy devices: a population-based cohort study using a nationwide database in Japan. International Journal of Surgery 202077 198-204. (https://doi.org/10.1016/j.ijsu.2020.03.072)

5 Burkey SH, Van Heerden JA, Thompson GB, Grant CS, Schleck CD \& Farley DR. Reexploration for symptomatic hematomas after cervical exploration. Surgery 2001130 914-920. (https://doi.org/10.1067/ msy.2001.118384)

6 Merki V, Pichler J, Giger R \& Mantokoudis G. Chylothorax in thyroid surgery: a very rare case and systematic review of the literature. Journal of Otolaryngology: Head and Neck Surgery 201645 52. (https://doi. org/10.1186/s40463-016-0166-y)

7 Weiss A, Lee KC, Brumund KT, Chang DC \& Bouvet M. Risk factors for hematoma after thyroidectomy: results from the nationwide inpatient sample. Surgery 2014156 399-404. (https://doi.org/10.1016/j. surg.2014.03.015)

8 Campbell MJ, McCoy KL, Shen WT, Carty SE, Lubitz CC, Moalem J, Nehs M, Holm T, Greenblatt DY, Press D, et al. A multi-institutional international study of risk factors for hematoma after thyroidectomy. Surgery 2013154 1283-1289; discussion 1289. (https://doi. org/10.1016/j.surg.2013.06.032)

9 Godballe C, Madsen AR, Sørensen CH, Schytte S, Trolle W, HelwegLarsen J, Barfoed L, Kristiansen L, Sørensen VZ, Samuelsen G, et al. Risk factors for recurrent nerve palsy after thyroid surgery: a national study of patients treated at Danish Departments of ENT Head and Neck Surgery. European Archives of Oto-Rhino-Laryngology 2014271 2267-2276. (https://doi.org/10.1007/s00405-013-2767-7)

10 Bergenfelz A, Jansson S, Kristoffersson A, Mårtensson H, Reihnér E, Wallin $\mathrm{G} \&$ Lausen I. Complications to thyroid surgery: results as reported in a database from a multicenter audit comprising 3,660 patients. Langenbeck's Archives of Surgery 2008393 667-673. (https:// doi.org/10.1007/s00423-008-0366-7)

11 Bergenfelz A, Salem AF, Jacobsson H, Nordenström E, Almquist M \& Steering Committee for the Scandinavian Quality Register for Thyroid, Parathyroid and Adrenal Surgery (SQRTPA). Risk of recurrent laryngeal nerve palsy in patients undergoing thyroidectomy with and without intraoperative nerve monitoring. British Journal of Surgery 2016103 1828-1838. (https://doi. org $/ 10.1002 /$ bjs.10276

12 Mullen JT, Davenport DL, Hutter MM, Hosokawa PW, Henderson WG, Khuri SF \& Moorman DW. Impact of body mass index on perioperative outcomes in patients undergoing major intra-abdominal cancer surgery. Annals of Surgical Oncology 200815 2164-2172. (https://doi. org/10.1245/s10434-008-9990-2)

13 Mullen JT, Moorman DW \& Davenport DL. The obesity paradox: body mass index and outcomes in patients undergoing nonbariatric general surgery. Annals of Surgery 2009250 166-172. (https://doi.org/10.1097/ SLA.0b013e3181ad8935)

14 Mathur AK, Ghaferi AA, Osborne NH, Pawlik TM, Campbell DA, Englesbe MJ \& Welling TH. Body mass index and adverse perioperative outcomes following hepatic resection. Journal of Gastrointestinal Surgery 201014 1285-1291. (https://doi.org/10.1007/s11605-010-1232-9)

15 Yasunaga H, Horiguchi H, Matsuda S, Fushimi K, Hashimoto H \& Ayanian JZ. Body mass index and outcomes following gastrointestinal cancer surgery in Japan. British Journal of Surgery 2013100 1335-1343. (https://doi.org/10.1002/bjs.9221)

16 Ri M, Miyata H, Aikou S, Seto Y, Akazawa K, Takeuchi M, Matsui Y, Konno $\mathrm{H}$, Gotoh M, Mori M, et al. Effects of body mass index (BMI) on surgical outcomes: a nationwide survey using a Japanese web-based database. Surgery Today 201545 1271-1279. (https://doi.org/10.1007/ s00595-015-1231-2)
17 Konishi T, Fujiogi M, Michihata N, Morita K, Matsui H, Fushimi K, Tanabe M, Seto Y \& Yasunaga H. Impact of body mass index on outcomes after breast cancer surgery: nationwide inpatient database study in Japan. Clinical Breast Cancer 202020 e663-e674. (https://doi org/10.1016/j.clbc.2020.05.002)

18 Buerba R, Roman SA \& Sosa JA. Thyroidectomy and parathyroidectomy in patients with high body mass index are safe overall: analysis of 26,864 patients. Surgery $2011150950-958$. (https://doi.org/10.1016/j.surg.2011.02.017)

19 Milone M, Musella M, Conzo G, Campana G, De Filippo D, Coretti G Amato M, Salvatore G, Amato B \& Milone F. Thyroidectomy in high body mass index patients: a single center experience. International Journal of Surgery 201628 (Supplement 1) S38-S41. (https://doi. org/10.1016/j.ijsu.2015.12.054)

20 Finel JB, Mucci S, Branger F, Venara A, Lenaoures P, Rodien P \& Hamy A. Thyroidectomy in patients with a high BMI: a safe surgery? European Journal of Endocrinology 2014 171 99-105. (https://doi. org/10.1530/EJE-14-0063)

21 Blanchard C, Bannani S, Pattou F, Brunaud L, Hamy A, Christou N, Mathonnet M, Dahan M, Prades JM, Landecy G, et al. Impact of body mass index on post-thyroidectomy morbidity. Head and Neck 201941 2952-2959. (https://doi.org/10.1002/hed.25773)

22 Greenland S. Dose-response and trend analysis in epidemiology: alternatives to categorical analysis. Epidemiology 19956 356-365. (https://doi.org/10.1097/00001648-199507000-00005)

23 Greenland S. Avoiding power loss associated with categorization and ordinal scores in dose-response and trend analysis. Epidemiology 19956 450-454. (https://doi.org/10.1097/00001648-199507 000-00025)

24 Yasunaga H. Real world data in Japan: chapter II the diagnosis procedure combination database. Annals of Clinical Epidemiology 2019 1 76-79. (https://doi.org/10.37737/ace.1.3_76)

25 Yamana H, Moriwaki M, Horiguchi H, Kodan M, Fushimi K \& Yasunaga H. Validity of diagnoses, procedures, and laboratory data in Japanese administrative data. Journal of Epidemiology 201727 476-482. (https://doi.org/10.1016/j.je.2016.09.009)

26 Urano T, Miyauchi A, Shimizu K, Tomoda C, Takamura Y, Ito Y, Miya A, Kobayashi K, Matsuzuka F, Amino N, et al. A prophylactic infusion of calcium solution reduces the risk of symptomatic hypocalcemia in patients after total thyroidectomy. World Journal of Surgery 200630 304-308. (https://doi.org/10.1007/s00268-005-0374-5)

27 Sanabria A, Dominguez LC, Vega V, Osorio C \& Duarte D. Routine postoperative administration of vitamin $\mathrm{D}$ and calcium after total thyroidectomy: a meta-analysis. International Journal of Surgery 20119 46-51. (https://doi.org/10.1016/j.ijsu.2010.08.006)

28 Charlson ME, Pompei P, Ales KL \& MacKenzie CR. A new method of classifying prognostic comorbidity in longitudinal studies: development and validation. Journal of Chronic Diseases 198740 373-383. (https://doi.org/10.1016/0021-9681(87)90171-8)

29 Ito Y, Onoda N \& Okamoto T. The revised clinical practice guidelines on the management of thyroid tumors by the Japan Associations of Endocrine Surgeons: core questions and recommendations for treatments of thyroid cancer. Endocrine Journal 202067 669-717. (https://doi.org/10.1507/endocrj.EJ20-0025)

30 WHO Expert Consultation. Appropriate body-mass index for Asian populations and its implications for policy and intervention strategies. Lancet 2004363 157-163. (https://doi.org/10.1016/S01406736(03)15268-3)

31 OECD. Overweight or obese population (indicator), 2021. Accessed 1 February 2021. (https://doi.org/10.1787/86583552-en)

32 Roger VL. Epidemiology of heart failure. Circulation Research 2013113 646-659. (https://doi.org/10.1161/CIRCRESAHA.113.300268)

33 El-Orbany M \& Woehlck HJ. Difficult mask ventilation. Anesthesia and Analgesia 2009109 1870-1880. (https://doi.org/10.1213/ ANE.0b013e3181b5881c)
This work is licensed under a Creative Commons Attribution-NonCommercial 4.0 International Ljcensen Bioscientifica.com at 04/26/2023 10:40:10AM 
34 Casati A \& Putzu M. Anesthesia in the obese patient: pharmacokinetic considerations. Journal of Clinical Anesthesia 200517 134-145. (https://doi.org/10.1016/j.jclinane.2004.01.009)

35 Procter LD, Davenport DL, Bernard AC \& Zweischenberger JB. General surgical operative duration is associated with increased risk-adjusted infectious complication rates and length of hospital stay. Journal of the American College of Surgeons 2010210 60.e1-65.e1. (https://doi. org/10.1016/j.jamcollsurg.2009.09.034)

36 Davenport DL, Xenos ES, Hosokawa P, Radford J, Henderson WG \& Endean ED. The influence of body mass index obesity status on vascular surgery 30-day morbidity and mortality. Journal of Vascular Surgery 200949 140-147, 147.e1; discussion 147. (https://doi. org/10.1016/j.jvs.2008.08.052)

37 Kitagawa W, Shimizu K, Akasu H \& Tanaka S. Endoscopic neck surgery with lymph node dissection for papillary carcinoma of the thyroid using a totally gasless anterior neck skin lifting method. Journal of the American College of Surgeons 2003196 990-994. (https://doi. org/10.1016/S1072-7515(03)00130-3)

38 Kuboki A, Nakayama T, Konno W, Goto K, Nakajima I, Kanaya H, Hirabayashi H \& Haruna S. New technique using an energy-based device versus conventional technique in open thyroidectomy. Auris, Nasus, Larynx 201340 558-562. (https://doi.org/10.1016/j.anl.2013.04.005)

39 Maeda H, Kutomi G, Satomi F, Shima H, Mori M \& Takemasa I. Comparison of surgical outcomes and complications between the harmonic FOCUS and conventional surgery for open thyroidectomy. Molecular and Clinical Oncology 20188 553-556. (https://doi. org/10.3892/mco.2018.1569)

40 OECD. Length of hospital stay (indicator), 2021. Accessed 1 February 2021. (https://doi.org/10.1787/8dda6b7a-en)

41 Muramatsu N \& Liang J. Hospital length of stay in the United States and Japan: a case study of myocardial infarction patients. International Journal of Health Services: Planning, Administration, Evaluation 199929 189-209. (https://doi.org/10.2190/8A4W-83KG-J5MU-CVV2)

42 Kuba S, Yamanouchi K, Hayashida N, Maeda S, Adachi T, Sakimura C, Kawakami F, Yano H, Matsumoto M, Otsubo R, et al. Total thyroidectomy versus thyroid lobectomy for papillary thyroid cancer: comparative analysis after propensity score matching: a multicenter study. International Journal of Surgery 201738 143-148. (https://doi.org/10.1016/j.ijsu.2016. 09.083)

Received in final form 22 September 2021

Accepted 30 September 2021

Accepted Manuscript published online 30 September 2021 (c) 2022 The authors Published by Bioscientifica Ltd.
This work is licensed under a Creative Commons Attribution-NonCommercial 4.0 International dedcensen Bioscientifica.com at 04/26/2023 10:40:10AM 